\title{
Der Mensch als landschaftsprägender Faktor des westlichen Bodenseegebietes seit dem späten Atlantikum
}

\author{
MANFRED RÖSCH *)
}

\author{
Prehistory, Atlanticum, Neolithic, Bronze age, pollen diagrams, \\ macrofossil analysis, human colonisation, interrelationship, man-landscape \\ Lake Constance region, Baden-Württemberg \\ TK 25: Nr. 8119, 8120, 8219, 8220, 8221, 8319, 8320
}

\begin{abstract}
Kurzfassung: Eingebunden in ein längerfristiges archäologisches Projekt zur jungsteinzeitlichen und bronzezeitlichen Besiedlungsgeschichte im Alpenvorland tragen archäobotanische Untersuchungen zur Kenntnis der Wechselwirkung Mensch - Landschaft in dieser Zeit bei. Pollenanalysen in Toteislöchern und in der Flachwasserzone des Bodensees sowie Großrestanalysen an den Kulturschichten praehistorischer Feuchtbodensiedlungen geben Hinweise darauf, daß bereits ab dem späten Atlantikum ein enger Zusammenhang zwischen menschlicher Besiedlung und Wirtschaft und der Veränderung terrestrischer und limnischer Ökosysteme bestand.
\end{abstract}

[Environmental Changes caused by Man since Late Atlantic Time at Western Lake Constance]

Abstract: Archeobotanical investigations are associated with a long-dated archeological project dealing with history of human colonisation during Neolithic time and Bronze age in South western Germany. These investigations enlarge the knowledge of human inpact on environment and viceversa. Pollen Diagrams from kettle holes and from lake shore sediments of western lake Constance together with macrofossil analysis from cultural layers of Neolithic lake shore dwellings give reason of the fact, that already since Late Atlantic there was a close connection between human colonisation and economics and changes of terrestric and limnic environments.

\section{Einleitung}

Das 1983 ins Leben gerufene DFG-Schwerpunktprogramm ,Siedlungsarchäologische Untersuchungen im Alpenvorland" will mittels großangelegter Siedlungsgrabungen und integrierter naturwissenschaftlicher

*) Anschrift des Autors: Dr. M. RÖsCH, Institut für Urund Frühgeschichte der Universität Freiburg, Fischersteig 9, D -7766 Hemmenhofen.
Untersuchungen den seit den Dreißiger Jahren in Deutschland stagnierenden Kenntnisstand über Struktur und Wirtschaft der sogenannten „Pfahlbauten", ihre Einbindung in den Naturraum und dessen Veränderung den Möglichkeiten moderner Methoden anpassen (SCHLICHTERLE 1985a). Eine Schlüsselrolle kommt hierbei der Archäobotanik zu, die einerseits durch Untersuchung der Pflanzenreste aus Kulturschichten zur Kenntnis von Ernährung und Wirtschaft in vorgeschichtlicher Zeit beiträgt, andererseits durch Pollenanalysen stratigraphische Korrelationsmöglichkeiten, sowie Einblick in die regionale Vegetationsund Landschaftsgeschichte bietet. Durch absolute Chronologie mittels Dendrochronologie und kalibrierter Radiocarbondaten können kulturelle und landschaftliche Veränderungen in zeitliche, durch den Vergleich zwischen Nutzung und Vegetationsveränderung in kausale Beziehung gebracht werden.

Der Referenz-Naturraum (BERGLUND 1979) westliches Bodenseegebiet weist infolge würmzeitlicher Vergletscherung zahlreiche Hohlformen auf und wurde im Hegau seit der Bandkeramik, am Seeufer seit dem Jungneolithikum von Bauernkulturen besiedelt (vgl. Abb. 1). Die vorliegenden botanischen Untersuchungen an Kulturschichten konzentrieren sich auf den Siedlungsplatz Hornstaad-Hörnle mit seinen 5 bekannten, teilweise mehrphasigen Anlagen des Jungund Endneolithikums (DIECKMANN 1985; RÖSCH 1985 c). Daneben gibt es orientierende und allesamt unpublizierte Untersuchungen in jungneolithischen Stationen von Nußdorf (S. GREGG) und Wallhausen (M. RÖSCH), sowie in endneolithischen Stationen von Sipplingen (St. JACOMET), Allensbach (S. KARG, St. JACOMET und M. RÖSCH) und Wallhausen (M. RösCH). Die Grundzüge der regionalen Vegetationsgeschichte im Holozän sind durch Pollenanalysen am Mindelsee (LANG 1970, 1973, 1983) und an den Nussbaumer Seen (RÖSCH 1983, 1985a) bekannt. 


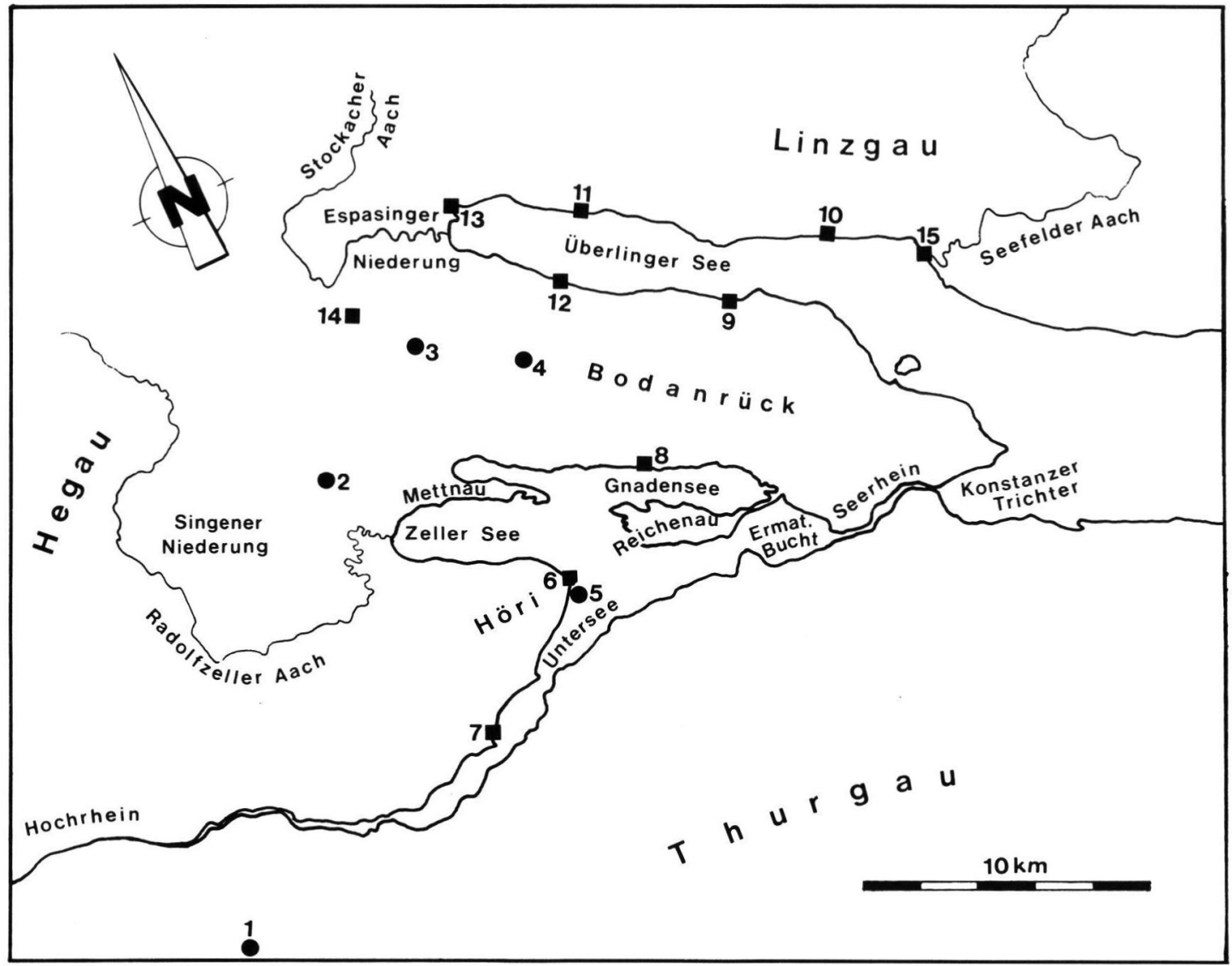

- Archäobotanische Untersuchungen in jungsteinzeitlichen

- Feuchtbodensiedlungen

Seen / Moor mit Standardpollendiagramm

1 Nussbaumer Seen

9 Wallhausen

2 Feuenried

10 Nußdorf

3 Durchenbergried

11 Sipplingen

4 Mindelsee

12 Bodman-Blissenhalde

5 HoB 1

13 Bodman-Schachen

6 Hornstaad-Hörnle

14 Stahringen

7 Wangen

15 Hagnau

8 Allensbach

Abb. 1: Übersicht Paläoökologie westlicher Bodensee.

Abb. 1 zeigt die Lage dreier Standardpollenprofile im Feuenried bei Überlingen am Ried, im Durchenbergried bei Güttingen und bei Hornstaad-Hörnle. Im Durchenbergried, einem kleinen, ehemaligen Toteisloch, wird der auskleidende Beckenton von kalkfreien Mudden und mesotrophen Torfen mit insgesamt bis 10 m Mächtigkeit überlagert (RÖSCH 1986). Die Flachwasserzone bei Hornstaad, bis $300 \mathrm{~m}$ breit, besteht aus fein geschichtetem Kalksilt, der in Mächtigkeiten bis $14 \mathrm{~m}$ dem Beckenton aufliegt und an der unter- suchten Stelle ein lückenloses Holozänprofil darstellt. An beiden Orten sind Untersuchungen im Gang. Im Feuenried erlaubt ein Prozent- und Influxdiagramm an einem 5,5 m mächtigen Profil mit der Abfolge Beckenton - kalkfreie Mudde - Niedermoortorf, gestützt auf $33{ }^{14} \mathrm{C}$-Daten, weitreichende Aussagen zur holozänen Stratigraphie und Vegetationsgeschichte (RÖSCH 1985 b, dort auch Hinweise zur Methodik). Die Geschichte des Sedimentationsraumes ist bei RÖSCH (1986) dargestellt. 


\section{Darstellung}

Zum Vergleich von Landschafts- und Besiedlungsgeschichte ist die Umstellung von konventioneller zu kalibrierter Chronologie notwendig. In Abb. 2 sind deshalb der jüngere Teil des Pollenprofils Feuenried ab dem Mittleren Atlantikum entlang der kalibrierten Zeitachse dargestellt (Kalibration nach STUIVER 1982, Gallay \& al. 1983, Pearson \& al. 1983, Kromer \& al. 1985 oder KIEIN \& al. 1982). Dieser Skala vorangestellt sind die Chronozonen sensu MANGERUD \& al. (1974). Das Diagramm zeigt neben den prozentualen Anteilen (Linienkurven, im Hauptdiagramm Flächen und Signaturkurven) die Influxwerte (subfossiler Pollenniederschlag pro Jahr und Fläche, als horizontale Balken). Die Einteilung bestimmter Pollentypen zu den Sammelgruppen als Zeiger für Ackerbau, Grünland, Waldweide und Ruderalstandorte orientiert sich an BEHRE (1981). Das gemeinsame Auftreten von Kulturzeigern (besonders Cerealia-Pollentyp) und typischen Veränderungen in den Gehölzpollenspektren veranlaßte die Abgliederung von 11 Rodungsphasen ( $\mathrm{R} 1$ bis R11). Dazwischen treten Sukzessionsphasen auf, die bisweilen ein Naturwaldstadium erreichen. Die Rodungsphasen können in ihrer Zeitstellung mit den dendrochronologisch ermittelten Schlagperioden der Pfahlbauhölzer am Bodensee (BILLAMBOZ \& BECKER 1985) und mit der Kulturgeschichte der Region verglichen werden.

\section{Kurzer Überblick über 7000 Jahre Landschafts- und Siedlungsgeschichte}

\section{(Vgl. hierzu Abb. 2 und Tab. 1)}

Die neolithische Erschließung der Region beginnt im mittleren Atlantikum an der westlichen Peripherie, im Hegau, wo 1985 in linearbandkeramischen Siedlungen im Umkreis des Hohentwiel ausgegraben wurde. Die Rodungsphasen R 1 und R 2 in Abb. 2 könnten hiermit in Zusammenhang stehen, doch sei betont, daß weder die Kulturzeigerwerte noch die Veränderungen der Gehölzspektren so kräftig sind, um allein neolithische Eingriffe in die Landschaft zu beweisen. Dies gilt auch für Rodungsphase R 3, die wohl mit der ebenfalls archäologisch belegten mittelneolithischen (RÖSSENER s. 1.) Siedlungstätigkeit im Hegau in Beziehung steht. Alle 3 Phasen fallen ins 6. Jahrtausend v. Chr., in collinen bis submontanen Lagen herrschte der Eichenmischwald vor. Bei der geschlossenen Buchenkurve könnte es sich um Fernflug aus Buchenbeständen montaner Lagen am Alpenrand handeln. Ihr Anstieg während der Rodungsphasen wäre dann eine Folge der Auflichtung des Eichenmischwaldes, wie dies auch für die Hasel gilt, deren Pollen jedoch nicht dem Fernflug entstammen dürfte, sondern lokalem Vorkommen im Unterstand. Das Feuenried liegt an der Nahtstelle zwischen Bodenseebecken und fluvioglazialen Schottern der Singener Niederung im Vorfeld des Hegau, $3 \mathrm{~km}$ westlich vom aktuellen Seeufer und $7 \mathrm{~km}$ östlich vom Hohentwiel.

Die erste rein palynologisch abgesicherte Rodungsphase, R4, durch ein Holzkohleband in Randlage des Feuenrieds in biostratigraphisch identischer Position als lokales Ereignis belegt, fällt in die 2. Hälfte des 5 . Jahrtausends deutlich vor die archäologisch belegte jungneolithische Besiedlung der Seeufer. Konventionell entspricht dies dem jüngeren Atlantikum (Mitte). Biostratigraphisch ist soeben der Anstieg der Buchenkurve zur Kodominanz mit dem EMW erfolgt und der Ulmen-Linden-Fall beginnt sich abzuzeichnen. Aufgrund mehrerer Daten kann man den Beginn der rationalen Buchenkurve im Rheingletschergebiet am Beginn des späten Atlantikums um 6000 BP annehmen. Für diese plötzliche massive Buchenausbreitung nach mehr als tausendjähriger Präsenz mit geringer Bedeutung sind verschiedene Erklärungen denkbar. Neben klimatischen Ursachen (Frosnitz-Schwankung) kann an menschlichen Einfluß gedacht werden. Dieser kann mittelbar gewesen sein, beispielsweise durch Veränderung des Wildbestandes.

Während das Feuenried in Vorpostenlage die wohl noch mittelneolithische Bevölkerungsbewegung in die Jungmoräne bezeugt und die Rodungsphase R 5 hier nur eine Fortsetzung des bereits Dagewesenen darstellt, verkörpert die jungneolithische Besiedlung der Seeufer ab etwa $4000 \mathrm{BC}$ dort einen gravierenden Einschnitt, der im Diagramm klar zutage tritt (Abb. 3): Mit dem Auftreten einer geschlossenen und recht massiven Kulturzeigerkurve, durch biostratigraphische Korrelation um $4000 \mathrm{BC}$ anzusetzen, fallen die Kurven von Buche und EMW, besonders der Ulme, abrupt ab, die Hasel wird dominant und die Pollenkonzentration sinkt auf ein Viertel, wohl als Folge erhöhter Sedimentwachstumsrate. Die natürliche zonale Vegetation jener Zeit war wohl schon deutlich buchendominiert, die EMW-Elemente kann man sich eher reliktisch eingesprengt oder azonal an Auestandorten denken. Der vorübergehende Rückgang der Buche während des Jungneolithikums und der endgültige von Ulme und Linde muß in Zusammenhang mit der menschlichen Siedlungstätigkeit gesehen werden. Am Bodenseeufer kann aufgrund archäologischer Belege ab dem Jungneolithikum von einer hohen Siedlungsdichte mit Distanzen von vielleicht $5 \mathrm{~km}$ zwischen zeitgleichen Anlagen ausgegangen werden (SCHLICHTHERLE 1985b). Im Hinterland erlaubt der Forschungsstand keine Aussagen. Ungeheure Mengen von verbautem Holz, als Pfähle und liegende Hölzer erhalten und der dendrologischen Forschung zugänglich, manifestieren nur einen Teil 
FEUENRIED BEI UBERLINGEN AM RIED Prozent/Influx-kalibrierte Zeitachse

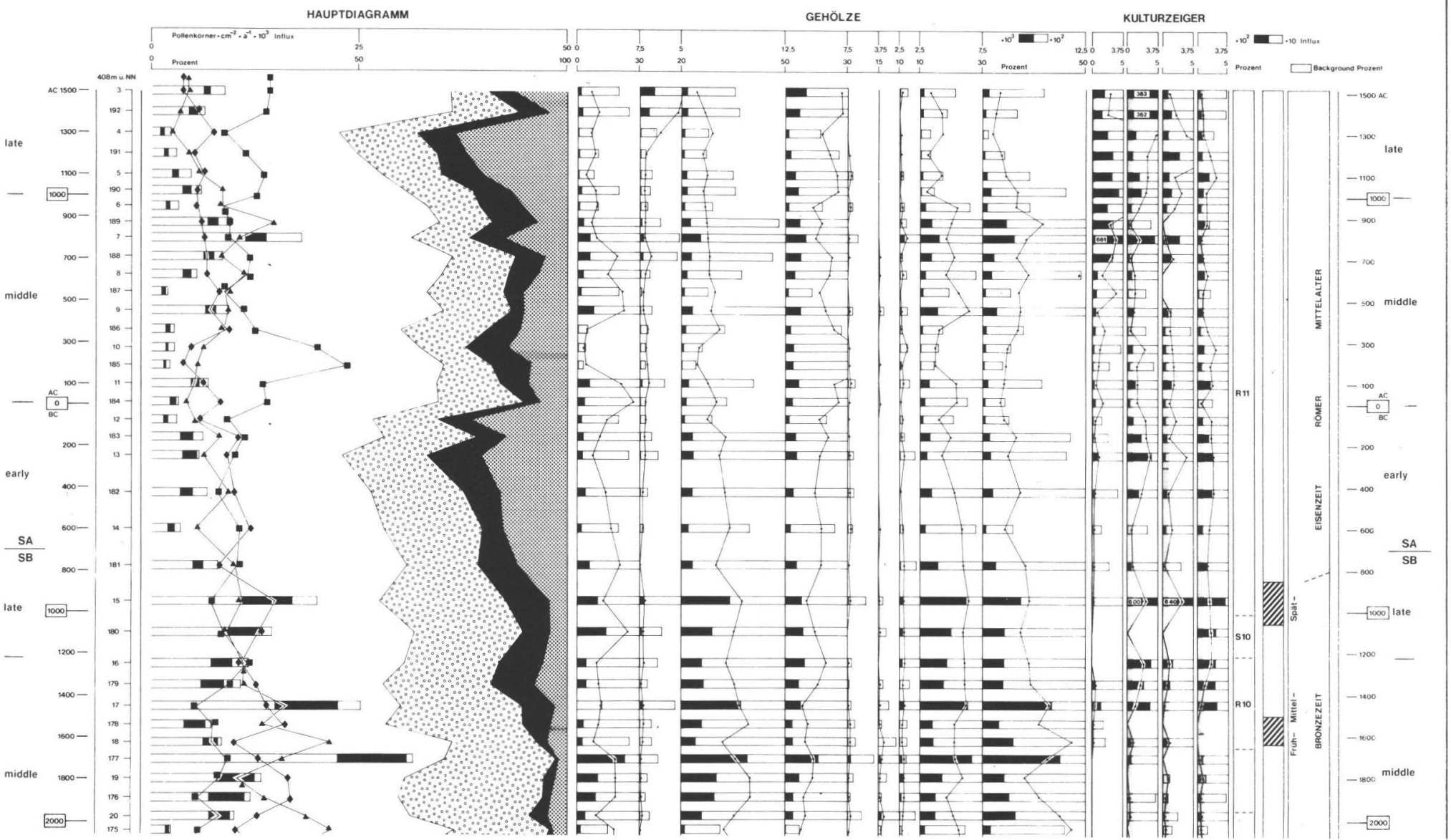




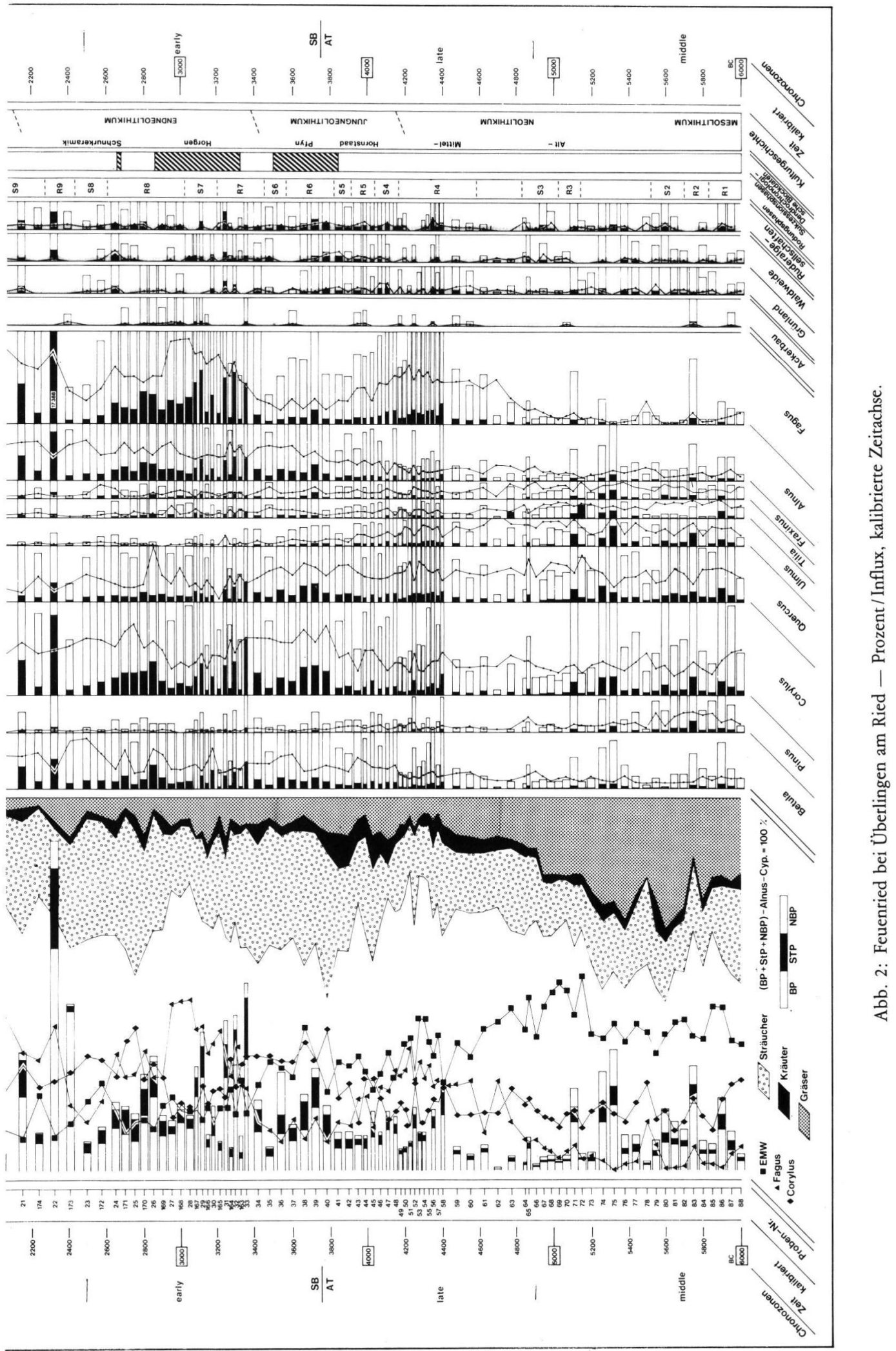


Tab. 1: Synopsis prähistorischer Kultur- und Landschaftsgeschichte im westlichen Bodenseegebiet

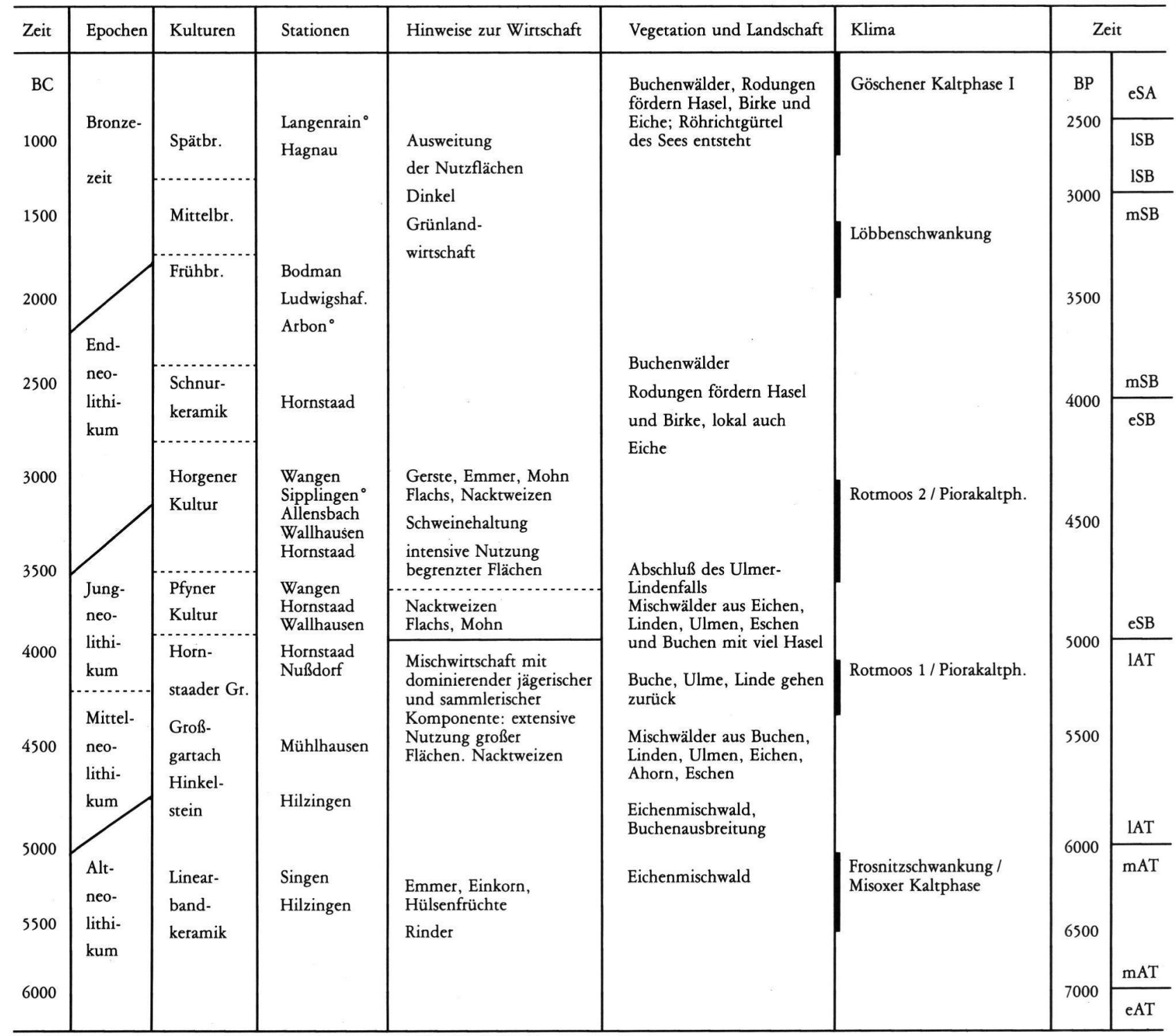



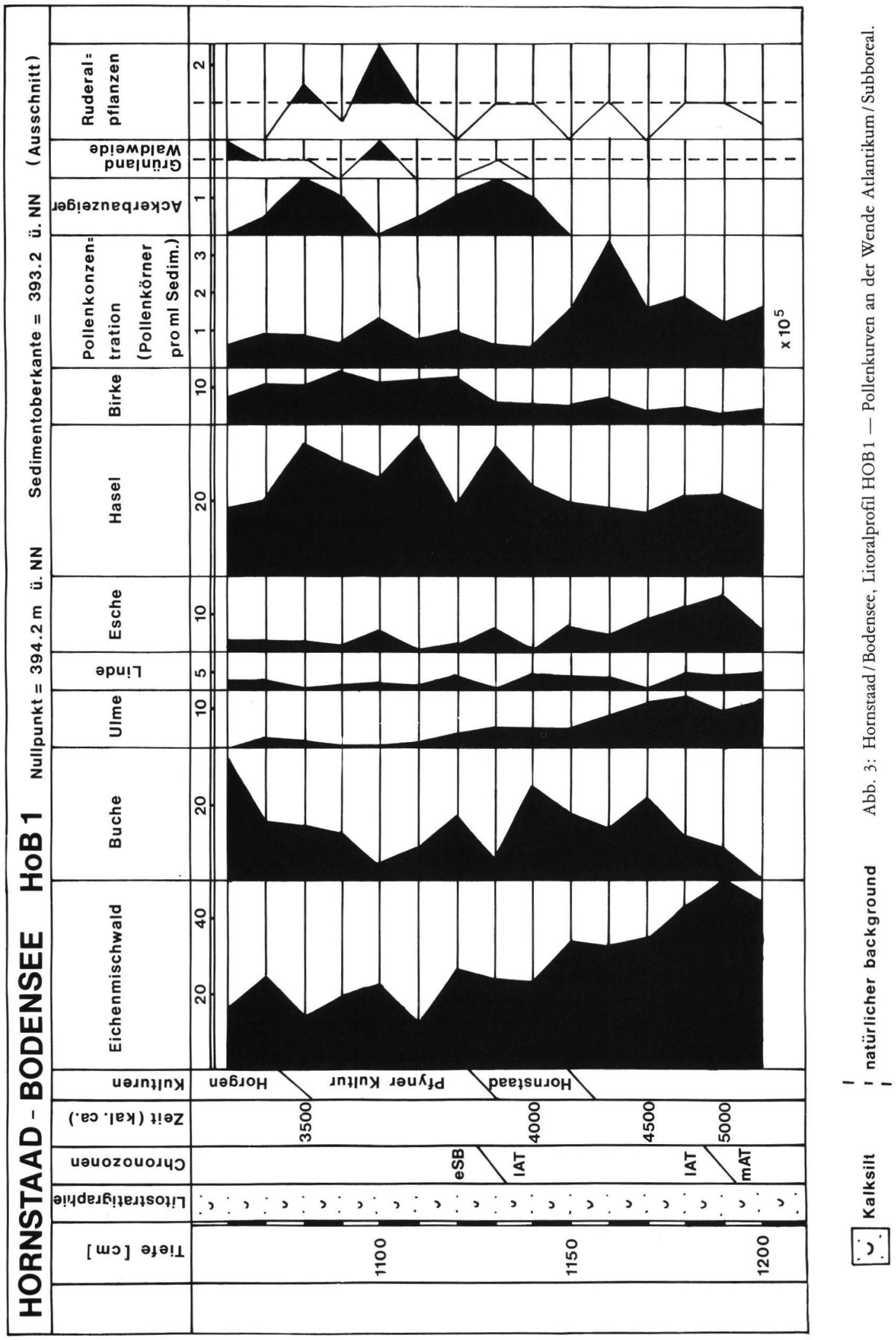
der Holznutzung, zumal die laut Pollendiagramm besonders in Mitleidenschaft gezogenen Arten hier wenig beteiligt sind. Der Brennholzbedarf ist sicher nicht zu unterschätzen und kann mittels Holzkohlebestimmung mindestens qualitativ erfaßt werden. Weitere Nutzungen, für die bereits Hinweise vorliegen, sind Bastgewinnung (hier ist vor allem an die Linde zu denken) sowie Laubheufütterung, nachweisbar durch Zweige und Knospenschuppen und denkbar für weichlaubige Arten wie Ulme, Linde, Buche, Esche, zumal Tierhaltung durch Knochenanalyse belegt ist (KOKABI 1985), es aber noch kein Grünland gab. Im Samenspektrum der Kulturschichten des frühen Jungneolithikums (Hornstaader Gruppe) spielen neben den Kulturpflanzen Nacktweizen (Triticum aestivum/durum, vorherrschend), Gerste (Hordeum vulgare L.), Emmer (Triticum dicoccum) SCHRANK, Einkorn (Triticum monococcum L.), Flachs (Linum usitatissimum L.) und Schlafmohn (Papaver somniferum L.) wild gesammelte Früchte und Samen eine große Rolle. Hier treten neben Haselnüssen (Corylus avellana L.) und Bucheckern (Fagus sylvatica L.), Erdbeere (Fragaria vesca L.), Himbeere (Rubus idaeus L.), Erdbeere (Fragaria vesca L.), Himbeere (Rubus idaeus L.), Brombeere (Rubus fruticosus agg.), Kratzbeere (Rubus caesius L.), Rote Kornellkirsche Cornus sanguinea L.), Schlehe (Prunus spinosa agg.), Traubenkirsche (Prunus padus L.), Hagebutte (Rosa cf. canina agg.), Judenkirsche (Physalis alkekengi L.), Äpfeln (Malus cf. sylvestris agg.) und anderen Nüssen und Obst auch heute nicht mehr genutzte Samen auf, wie die des Stechenden Hohlzahns (Galeopsis tetrahit agg.), des Feldkohls (Brassica rapa ssp. campestris (L.) Claph.), des Sophienkrauts (Descurainia sophia (L.) Webb) und des Leindotters Camelina sativa (L.) Crantz), deren Nutzung durch Vorratsfunde belegt ist.

Zusammen mit der Tatsache, daß die entsprechenden Knochenspektren überwiegend von Wildtieren stammen (KOKABI 1985), spricht dies für eine Mischwirtschaft mit vorherrschender sammlerischer und jägerischer Komponente in der Initialphase des alpenländischen „Pfahlbauneolithikums”, jedenfalls am Bodensee. Daraus folgt eine Zweigliederung der Siedlungskammern in kleine Areale für Nahrungsproduktion in Dorfnähe und in ein großflächig zu wildbeuterischer Rohstoffgewinnung genutztes Hinterland. Dieses Hinterland könnte in dicht besiedelten Räumen die Naturwaldreserven mehr oder weniger vollständig erfaßt und zu den geschilderten Veränderungen im Waldbild geführt haben.

In Abb. 2 ist R 5 mit der Hornstaader Gruppe in Verbindung zu bringen, R 6 mit der Pfyner Kultur, die das vollentwickelte Jungneolithikum, etwa zwischen 3850 und $3500 \mathrm{BC}$, am Bodensee verkörpert. Wie die große Ähnlichkeit von Hornstaader Großrestspektren der späten Pfyner und der frühen Horgener Kultur belegt, vollzog sich in dieser Zeit, und zwar vor dem kulturellen Übergang zur endneolithischen Horgener Kultur, ein wirschaftlicher Umschwung, in dem die Kulturpflanzen, jetzt insbesondere Gerste, Emmer, Flachs und Mohn, stark an Bedeutung gewinnen auf Kosten wild gesammelter Pflanzen, eine Entwicklung, die auf dem Sektor tierischer Nahrungsmittel völlig parallel läuft (KOKABI, in Vorber.). Für eine gewaltige Ausweitung der Nahrungsmittelproduktion spricht auch das vermehrte Auftreten von Segetalund Ruderalarten, die Hinweise auf die Ausbildung von Hack- und Halmfrucht- sowie Leinunkrautgesellschaften geben. Als Beispiel für letztere steht die in Kulturschichten des späten Jung- und Endneolithikums am Bodensee mit großer Stetigkeit auftretende Kreta-Flachsnelke (Silene cretica L.), die erstmals von JACOMET (1985) am Zürichsee nachgewiesen wurde.

Für das späte Jungneolithikum liegen von Seiten der Dendroarchäologie erste Hinweise auf Stockwaldwirtschaft vor (BILLAMBOZ 1986). Rindenmoosspektren aus endneolithischen Kulturschichten - Waldmoose sind ein wichtiger Bestandteil prähistorischer Rohstoffwirtschaft - weisen im Vergleich zu denen des frühen Jungneolithikums einen höheren Anteil lichtbedürftiger Arten auf (RÖsCH, in Vorber.).

Diese Entwicklung legt eine Konzentration der wirtschaftlichen Aktivität auf die engere Umgebung der Siedlungen und ein Nachlassen oder Ausbleiben des wildbeuterischen Nutzungsdrucks im bewaldeten Hinterland der Siedlungen nahe, das in bereits gestörten Beständen nun zur alleinigen Dominanz der Schatthölzer führte, die sich in der ersten Buchenpraedominanz des Subboreals ab 3200 BC (Mitte ISB) manifestiert. In Gebieten mit hohem Weißtannenanteil, wie am Zürichsee (HEITZ-WENIGER 1978), verhält sich diese wie die Buche. Klimatische Steuerung dieser Schattholzausbreitung (Rotmoosschwankung 2) ist nicht auszuschließen.

Zwischen der letzten dendrochronologisch fixierten schnurkeramischen Ufersiedlung (Endneolithikum) und der ersten frühbronzezeitlichen Siedlung klafft eine zeitliche Lücke von fast 1000 Jahren, während der die Pollendiagramme zwar für geringe menschliche Eingriffe, nicht jedoch für ihr völliges Ausbleiben sprechen (Abb. 2, R9). Die dendrochronologische Lücke könnte den Forschungsstand widerspiegeln oder methodisch bedingt sein (dendrochronologisch datierbar sind nur Eichenhölzer mit einer Mindestzahl von Jahrringen). Sie könnte aber auch eine Erhaltungslücke darstellen oder demographische Ursachen haben. In den beiden letzten Fällen sind Verbindungen zur See-und letztlich zur Klimageschichte zu 
suchen. Klimaverschlechterungen für diese Zeit sind allerdings nicht bekannt.

Ein weiterer wirtschaftlicher Umschwung am Beginn der Frühbronzezeit, zu Beginn des 2. Jahrtausends $\mathrm{BC}$, wurde bisher nur pollenanalytisch erfaßt, da keine Großrestanalysen aus entsprechenden Kulturschichten am Bodensee vorliegen. Nun ist mit Wirtschaftsgrünland und damit mit einer starken Vergrößerung des Bedarfs an gerodeter Fläche zu rechnen (R13, R11).

Mit dem Ende der Spätbronzezeit bricht um 850 BC die Besiedlung der Seeufer, andernorts auch der Moore und Talauen $\mathrm{ab}$. Ein schlüssiger Beweis für alleinig hydrologische, mithin klimatische Ursachen (Göschener Kaltphase 1) steht noch aus. Demographische Ursachen sind unwahrscheinlich, da ab der Spätbronzezeit das Pollendiagramm ständig hohen menschlichen Nutzungsdruck zeigt, ja sogar die nahezu vollständige Verdrängung der naturnahen buchenbeherrschten Wälder durch Hudewälder und Stockwälder mit kurzen Umtriebszeiten belegt. Das typische Landschaftsbild des Mittelalters entstand im Gebiet also offenbar schon zu Beginn der Eisenzeit (Rückgang der Buchenkurve und des Gehölzpolleninflux, Durchbruch der relativen Eichenkurve zur Praedominanz).

Ein letztmaliges starkes Nachlassen des menschlichen Nutzungsdrucks nach der Römerzeit (etwa 300 bis 500 $\mathrm{AC}$ ) ist in Abb. 2, vielleicht infolge eines kleineren Hiatus, nur andeutungsweise zu sehen. Diese Phase und die nachfolgende Entwicklung des Mittelalters und der Neuzeit konnte an den Nussbaumer Seen vollständig nachgezeichnet werden (RösCH 1983).

\section{Zum menschlichen Einfluß auf den Gewässerhaushalt}

Für kleine Seen wie die Nussbaumer Seen besteht ein Zusammenhang zwischen intensiver Landwirtschaft und verstärkter Sedimentwachstumsrate *) durch Eutrophierung und Eintrag erodierten Bodenmaterials. Dieser Prozeß setzte spätestens mit der frühmittelalterlichen Landnahme ein und verstärkte sich bis zur Gegenwart. Prähistorische Einflüsse sind schwerer nachweisbar, da hierbei kolluvialer Materialeintrag, der sonst bei der Steigerung der Sedimentwachstumsrate die Hauptrolle spielt, wohl infolge noch intakter Pufferzonen zwischen See und Feldflächen keine Rolle spielte. Dennoch ist beispielsweise an den Nussbaumer Seen in der Folge praehistorischer Rodungs-

*) Unter Sedimentwachstumsrate wird — im Gegensatz zur Sedimentationsrate - das Verhältnis von Sedimentmächtigkeit zu Sedimentationszeit ohne Berücksichtigung von Sedimentkompression verstanden. phasen, die in Zusammenhang mit Siedlungen am dortigen Ufer stehen dürften, eine vorübergehende Steigerung der Sedimentwachstumsrate zu beobachten. Damit korrespondiert die Tatsache, daß die bis zum späten Atlantikum gebildeten litoralen Sedimente des Bodensees mit ihrer Armut an Litoralpflanzen-Diasporen eine äußerst spärliche Vegetation im Uferbereich bezeugen, wie es typisch ist für ein oligotrophes Gewässer, wogegen die den Kulturschichten aufliegenden Sedimente mit ihrem hohen Gehalt an Wasserpflanzendiasporen für reiche Ufervegetation sprechen, von mehr oder weniger oligotrophen Characeenrasen über großblättrige Laichkrautgesellschaften (vor allem Potamogeton perfolatus L. und lucens L.), Nixkrautrasen (Najas marina L. s. str., intermedia Wolfg., flexilis (Willd.) Rostk. \& Schm. und minor All.) bis zur Teichfadengesellschaft. In einigem Abstand von den Kulturschichten verschwinden dann die Anzeichen für die Existenz meso- bis eutropher Gesellschaften und es bleiben die Characeen-Oogonien. $\mathrm{Ob}$ nun das bessere Nährstoffangebot edaphisch bedingt war durch die Kulturschicht selbst (JACOMET 1985 ) oder gelöst im Wasser vorlag und später ins Sediment wanderte, sei dahingestellt. Eine - vorübergehende - Eutrophierung randlicher Bereiche auch größerer Gewässer im Zuge praehistorischer Uferbesiedlung ist durchaus denkbar. Sie erklärt jedoch nicht die ab 4000 BC plötzlich verdreifachte Sedimentwachstumsrate (Abb. 4), die wohl vornehmlich durch Vermehrung des klastischen Sedimentanteils bedingt sein dürfte. Die Herkunft dieses Materials und die Ursachen für seine Feinschichtung durch wechselnden klastischen und karbonatischen Anteil zu klären bleibt der sedimentologischen Bearbeitung vorbehalten.

Im benachbarten Hegau jedenfalls sind mächtige Kolluvien bekannt, deren Bildungsbeginn mit der bandkeramischen Besiedlung zusammenfällt, als eindrucksvolle Zeugen rodungsbedingter Bodenerosion. Einen weiteren Hinweis für ähnliche Zusammenhänge stellt in der Flachwasserzone des Bodensees verstärktes Sedimentwachstum ab dem frühen Mittelalter dar.

Die Eutrophierung betraf nicht nur das Sub-, sondern auch das Eulitoral: Vom Bodensee und von anderen großen Alpenrandseen (JACOMET 1985) liegen Hinweise vor, daß sich ausgedehnte Röhrichtgürtel frühestens seit der Bronzezeit gebildet haben. Oligotrophe Strandlingsrasen, die ihren Platz eingenommen haben könnten, sind allerdings palynologisch oder durch Großreste schwer nachzuweisen.

\section{Moore, Mensch und Klima}

Menschliche Tätigkeit seit dem Neolithikum hinterließ also ihre Spuren an den Seen, indem verstärkte 


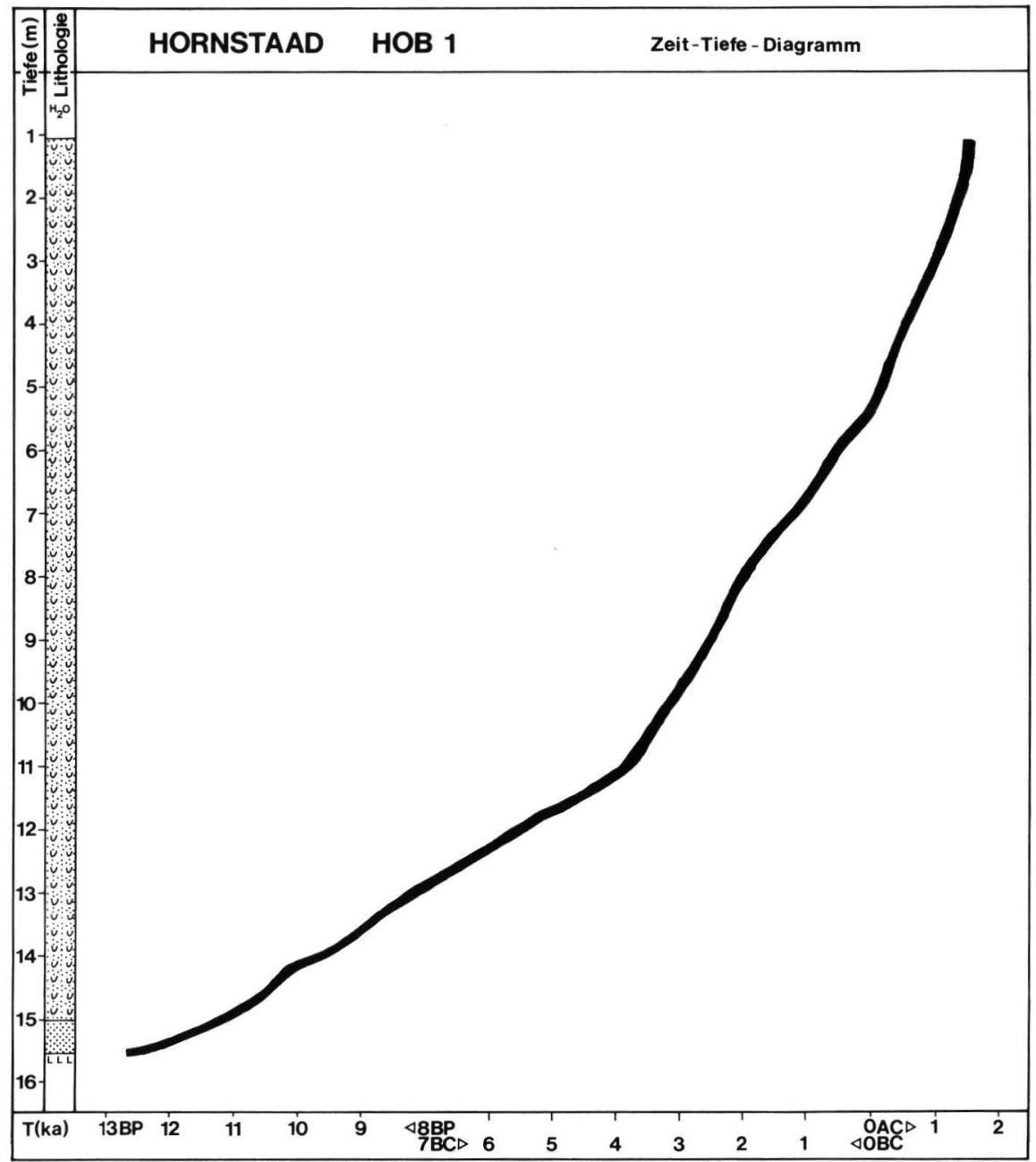

Abb. 4: Zeit-Tiefe-Diagramm des limnischen Profils Hornstaad HOB 1.

Abtragung das Erblinden kleiner Seen beschleunigte und bei kleinen die Flachwasserzone verbreiterte, in nicht ständig feuchten Hohlformen in Gestalt von Kolluvien und in Mooren in Form von Toneinschwemmungen. In den Zentren der Moore dürfte prähistorischer menschlicher Einfluß nur mit sehr empfindlichen Methoden nachweisbar sein (HÖLZER \& SCHLOSS 1981). Die Wachstumsschwankungen der im Gebiet untersuchten Moore scheinen synchron zu sein und mit den an der alpinen Waldgrenze nachgewiesenen Klimaverschlechterungen (BORTENSCHLAGER \& PATZELT 1969, ZOLLER \& al. 1966) zusammenzufallen. Zugleich stellen diese Abschnitte des Holozäns Zeiten kulturellen Übergangs dar. Auf diese Fragestellung wird die künftige Forschung ein besonderes Augenmerk richten, und besondere Bedeutung kommt dabei neben den Mooren dem Bodensee und seiner holozänen Pegelchronologie zu.

\section{Schriftenverzeichnis}

BEHRE, K.-E. (1981): The Interpretation of Anthropogenic Indicators in Pollen Diagrams. - Pollen et Spores, 23, 2: 225-245; Paris.

BERgLUnd, B. (ed.) (1979): Palaeohydrological changes in the Temperate Zone in the last 15000 years. Subproject B. Lake and mire environments, 1: 123 S. + 2 Appendices; Lund.

Billamboz, A. (1986): Zeitmesser Holz. Jahrringe erzählen Baugeschichte. - Archäologie in Deutschland, 1986; 1: 26-31; Stuttgart.

- \& BECKER, B. (1985): Dendrochronologische Eckdaten der neolithischen Pfahlbausiedlungen Südwestdeutschlands. - Ber. Ufer- u. Moorsiedlungen Südwestdeutschlands, 2. Materialh. Vor- u. Frühgesch. Bad.-Württ. 7: 80-97; Stuttgart. 
BORTenschlager, S. \& PAtzelt, G. (1969): Wärmezeitliche Klima- und Gletscherschwankungen im Pollenprofil eines hochgelegenen Moores $(2270 \mathrm{~m})$ der Venedigergruppe. - Eiszeitalter u. Gegenwart, 20: 116-122; Öhringen.

DieCKMANN, B. (1985): Die neolithischen Ufersiedlungen von Hornstaad-Hörnle am westlichen Bodensee. Die Grabungskampagne 1983/84. - Ber. z. Ufer- und Moorsiedlungen Südwestdeutschlands 2. Materialh. Voru. Frühgesch. in Bad.-Württ., 7, 98-124; Stuttgart.

Gallay, A., Olive, P. \& Carazetti, R. (1983): Chronologie $\mathrm{C} 14$ de la séquence Néolithique-Bronze ancien du Valais (Suisse). - Jb. Schweiz. Ges. Ur- u. Frühgesch., 66: 43-74; Frauenfeld.

Heitz-Weniger, A. (1978): Pollenanalytische Untersuchungen an den neolithischen und spätbronzezeitlichen Seerandsiedlungen „, Kleiner Hafner”, , ,Großer Hafner” und „Alpenquai" im untersten Zürichsee (Schweiz). Bot. Jb. Syst., 99; 1: 48-107; Stuttgart.

HÖLzeR, A. \& Schloss, S. (1981): Paläoökologische Studien an der Hornisgrinde (Nordschwarzwald) auf der Grundlage von chemischer Analyse, Pollen- und Großrestuntersuchung. - Telma, 11: 17-30; Hannover.

JACOMET, ST. (1985): Botanische Makroreste aus den Sedimenten des neolithischen Siedlungsplatzes AKAD-Seehofstraße am untersten Zürichsee. - Zürcher Studien zur Archäologie: 94 S. + Beilagen; Zürich (Juris).

Klein, J., Lerman, J., Damon, P. \& Ralph, E. (1982): Calibration of radiocarbon dates. - Radiocarbon, 24, 2: 103-150; New Haven, Con.

KOKABI, M. (1985): Vorläufiger Bericht über die Untersuchungen an Tierknochenfunden aus Hornstaad-Hörnle I am westlichen Bodensee. - Ber. Ufer- u. Moorsiedlungen Südwestdeutschlands 2. Materialh. Vor- u. Frühgesch. in Bad.-Württ., 7: 148-163; Stuttgart.

Kromer, B., Billamboz, A. \& Becker, B. (1985): Kalibration einer 100jährigen Baumringsequenz aus der Siedlung Aichbühl (Federsee). - Ber. Ufer- u. Moorsiedlungen Südwestdeutschl. 2. Materialh. Vor- u. Frühgesch. in Bad.-Württ., 7: 241-247; Stuttgart.

LANG, G. (1970): Florengeschichte und mediterran-mitteleuropäische Florenbeziehungen. - Feddes Repertorium, 81, 1-5: 315-335; Berlin.

- (1973): Die Vegetation des westlichen Bodenseegebiets. - Pflanzensoziologie, 17: 451 S.; Jena.

- (1983): Die Vegetation des Mindelsees und ihre Geschichte. In: Der Mindelsee. Die Natur- und Landschaftsschutzgebiete Baden-Württembergs, 11, 271-302; Karlsruhe.

Mangerud, J., Andersen, S. T., Berglund, B. \& Donner, J. (1974): Quaternary stratigraphy of Norden, a proposal for terminology and classification. - Boreas, 3: 109-128; Oslo.
Pearson, G., Pilcher, J. \& Baillie, M. (1983): High-precision $\mathrm{C} 14$ measurement of Irish oaks to show the natural C 14 variations from $200 \mathrm{BC}$ to $4000 \mathrm{BC}$. - Radiocarbon 25: 179-186; New Haven, Con.

Rösch, M. (1983): Geschichte der Nussbaumer Seen (Kanton Thurgau) und ihrer Umgebung seit dem Ausgang der letzten Eiszeit aufgrund quartärbotanischer, stratigraphischer und sedimentologischer Untersuchungen. - Mitt. Thurg. Naturf. Ges., 45: 110 S. + Beilagen; Frauenfeld.

- (1985a): Nussbaumer Seen - Spät- und postglaziale Umweltsveränderungen einer Seengruppe im östlichen Schweizer Mittelland. - In G. LANG (ed.): Swiss lake and mire environments during the last 15000 years. Diss. Bot, 87: 273-336; Vaduz.

- (1985 b): Ein Pollenprofil aus dem Feuenried bei Überlingen am Ried: Stratigraphische und landschaftsgeschichtliche Bedeutung für das Holozän im Bodenseegebiet. - Ber. Ufer- u. Moorsiedlungen Südwestdeutschlands 2: Materialh. Vor- u. Frühgesch. in Bad.Württ., 7: 43-79; Stuttgart.

- (1985c): Die Pflanzenreste der neolithischen Ufersiedlung von Hornstaad-Hörnle I am westlichen Bodensee. 1. Bericht. - Ber. Ufer- u. Moorsiedlungen Südwestdeutschl. 2: Materialh. z. Vor- u. Frühgesch. in Bad.Württ., 7: 164-199; Stuttgart.

- (1986): Zwei Moore des westlichen Bodenseegebiets als Zeugen prähistorischer Landschaftsveränderung. Telma 16 (im Druck).

SChlichtherle, H. (1985a): Ufer- und Moorsiedlungen zwischen Federsee und Bodensee. - In: SchreInER, A. \& METZ, B. (ed.): Exkursionsführer I: 14-34; Hannover (Deutsche Quartärvereinigung).

- (1985b): Prähistorische Ufersiedlungen am Bodensee - Eine Einführung in naturräumliche Gegebenheiten und archäologische Quellen. - Ber. Ufer- und Moorsiedlungen Südwestdeutschlands 2: Materialh. Vor- u. Frühgeschichte in Bad.-Württ. 7: 9-42; Stuttgart.

SCHREINER, A. (1973): Erläuterungen zu Blatt 8219 Singen der geologischen Karte von Baden-Württemberg $1: 25000 ; 130$ S. + Beilagen; Stuttgart.

Stuiver, M. (1982): A high-precision calibration of the $\mathrm{AD}$ radiocarbon time scale. - Radiocarbon 24, 1 : 1-26; New Haven, Con.

Zoller, H., Schindler, C. \& RÖTHLiSBerger, H. (1966): Postglaziale Gletscherstände und Klimaschwankungen im Gotthardmassiv und Vorderrheingebiet. - Verh. Naturf. Ges. Basel 77, 2: 97-164.

Manuskript eingegangen am 4. 8. 1986, Nachträge Oktober 1986. 
